\section{Artificial Intelligence for Engineering Design, Analysis and Manufacturing}

cambridge.org/aie

\section{Editorial}

Cite this article: Biswas $\mathrm{P}$, Orero $\mathrm{P}$, Metin Sezgin T (2019). Special issue on intelligent interaction design. Artificial Intelligence for Engineering Design, Analysis and Manufacturing 33, 369. https://doi.org/10.1017/

S0890060419000404

Author for correspondence:

Pradipta Biswas, E-mail: pb400@eng.cam.ac. uk

๑) Cambridge University Press 2019

\title{
Special issue on intelligent interaction design
}

Pradipta Biswas ${ }^{1}$, Pilar Orero ${ }^{2}$ and T. Metin Sezgin ${ }^{3}$ (Guest Editors)

${ }^{1}$ Indian Institute of Science, Banglore, India; ${ }^{2}$ University of Barcelona, Barcelona, Spain and ${ }^{3}$ Koc University, istanbul, Turkey

This issue of AI EDAM will discuss the application of artificial intelligence and related technologies for electronic user interface and interaction design. User interaction design plays a key role on the acceptance of any product, and advancement in artificial intelligence, computer vision, image processing, and other similar technologies made it possible to introduce new ways of interacting with machines. These intelligent user interfaces turn even more useful for people with different ranges of abilities and also for their able-bodied counterpart under situational impairment where the situation itself imposes constraint in terms of natural interaction. The issue covered topics like sketch recognition, computer vision, and AI algorithms for shape and object detection and accessibility in multimodal virtual reality environments through the following seven papers.

The first paper investigates sketching, which is a powerful natural tool for the product design process. The paper reviews existing technologies for free-hand sketch generation and interpretation for both $2 \mathrm{D}$ and $3 \mathrm{D}$ environments. The second paper presents a case study of developing a low-cost PCB (Printed Circuit Board) inspection tool and investigates computer vision algorithms to detect correct orientation and the type of IC (integrated circuit) chips under different lighting conditions. The third paper discusses computer vision and deep learning algorithms in further details for content analysis of live video in Hybrid TV. The paper proposes a customized description of visual information. The next paper investigates virtual reality environment which is gaining popularity in the smart manufacturing context as a form of Digital Twin providing immersive experience and undertook a study on positioning and guiding methods of subtitles in an immersive media. The last two papers report consider users with different range of abilities and present an interface to report quality of subtitles of visual information and cognitive games for users with cognitive impairment.

Overall, the issue covered topics are both directly related to product design and manufacturing as well as topics on accessibility which can, in turn, improve the inclusivity of the design and manufacturing process. 